\title{
Energetic Proton Generation in Ultra-Intense Laser-solid Interactions
}

S.C. Wilks, A.B. Langdon, T.E. Cowan, M. Roth, M. Singh, S. Hatchett, M.H. Key, D. Pennington, A. MacKinnon, R.A. Snavely

\section{March 1, 2000}

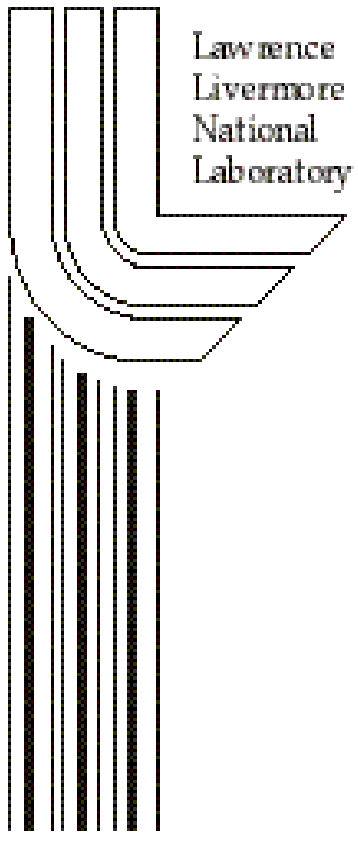




\section{DISCLAIMER}

This document was prepared as an account of work sponsored by an agency of the United States Government. Neither the United States Government nor the University of California nor any of their employees, makes any warranty, express or implied, or assumes any legal liability or responsibility for the accuracy, completeness, or usefulness of any information, apparatus, product, or process disclosed, or represents that its use would not infringe privately owned rights. Reference herein to any specific commercial product, process, or service by trade name, trademark, manufacturer, or otherwise, does not necessarily constitute or imply its endorsement, recommendation, or favoring by the United States Government or the University of California. The views and opinions of authors expressed herein do not necessarily state or reflect those of the United States Government or the University of California, and shall not be used for advertising or product endorsement purposes.

Work performed under the auspices of the U. S. Department of Energy by the University of California Lawrence Livermore National Laboratory under Contract W-7405-Eng-48.

This report has been reproduced

directly from the best available copy.

Available to DOE and DOE contractors from the

Office of Scientific and Technical Information

P.O. Box 62, Oak Ridge, TN 37831

Prices available from (423) 576-8401

http:/ /apollo.osti.gov/bridge/

Available to the public from the National Technical Information Service

U.S. Department of Commerce 5285 Port Royal Rd., Springfield, VA 22161

http://www.ntis.gov/

OR

Lawrence Livermore National Laboratory

Technical Information Department's Digital Library

http://www.llnl.gov/tid/Library.html 


\title{
Energetic Proton Generation in Ultra-Intense Laser-solid Interactions
}

\author{
S.C. Wilks, A.B. Langdon, T.E. Cowan, M. Roth, M. Singh, S. Hatchett, M.H. Key, D. Pennington, A. MacKinnon, and RA Snavely, \\ University of California, Lawrence Livermore National Laboratory, Livermore, CA 94550
}

\begin{abstract}
An explanation for the energetic ions observed in the PetaWatt experiments is presented. In solid target experiments with focused intensities exceeding $10^{20} \mathrm{~W} / \mathrm{cm}^{2}$, high-energy electron generation, hard bremsstrahlung, and energetic protons have been observed on the backside of the target. In this report, we attempt to explain the physical process present that will explain the presence of these energetic protons, as well as explain the number, energy, and angular spread of the protons observed in experiment. In particular, we hypothesize that hot electrons produced on the front of the target are sent through to the back off the target, where they ionize the hydrogen layer there. These ions are then accelerated by the hot electron cloud, to tens of $\mathrm{MeV}$ energies in distances of order tens of microns, whereupon they end up being detected in the radiographic and spectrographic detectors.
\end{abstract}

\section{INTRODUCTION}

The observation of energetic protons off the back of the gold and plastic millimeter-sized targets in the recent PetaWatt experiments has been the subject of great interest. Details of these experiments can be found in Key et. $\mathrm{al}^{1}$ and $\mathrm{S}$. Hatchett, et $\mathrm{al}^{2}$. This is mainly due to the fact that in these experiments, the currents have been estimated to be quite large. Measurements by Rich Snavely et $\mathrm{al}^{3}$, and M. Roth et al. ${ }^{4}$, have shown that a large number of protons are ejected off the back off the target. Taking this data, and normalizing with ITS, we estimate that about $10^{13}$ protons are ejected from the back. If one makes the assumption that the ions come off in a time of approximately 100 picoseconds, the current can be estimated to be of order MAmps. The energy spectrum has been measured two ways: directly with a spectrometer, ${ }^{4}$ and using various thickness filters in between sheets of radiochromic film ${ }^{3}$ that progressively stop the ions. The energy distribution shows an effective ion temperature of about $5 \mathrm{MeV}$, with the most energetic ions are around $50 \mathrm{MeV}$. The magnetic spectrometer gives a roughly power law spectrum for these ions. The spectrum is observed to be relatively flat, out to the cut-off energy, with the exception of the ion magnetic spectrometer, which shows a sharp increase in ion number at the highest ion energies. This monoenergetic feature will be described in two future publications. ${ }^{5}$

This paper will address two possible ion mechanisms associated with these experiments, and introduce a new and novel method of ion acceleration that is only possible with ultra-intense, short-pulse lasers. Briefly described, the new ion acceleration mechanism is the result of a "cloud" of hot electrons, 
generated in the blow-off plasma created by the laser prepulse interacting with the target, that go through the target and ionize the proton layer on the back of the target. These protons are then pulled off the surface by this cloud of electrons, and accelerated to tens of MeV's, in 10's of microns. Various aspects of the two mechanisms will be discussed with respect to the experiment. It is found that only the new method, henceforth referred to as Target Normal Sheath Acceleration (TNSA), is consistent with experimental findings.

We begin by reviewing previous research on energetic ion production using intense lasers, paying particular attention to the maximum energy gain, efficiency, and directionality. We then introduce a related but new acceleration mechanism, and present a 1-D model that explains some aspects of the acceleration. We then show where certain components of the 1-D model breaks down, due to the nonconstant nature of the electron temperature. We then show what happens when 2-D effects of the electrons spatial distribution is taken into account. Finally, once the physics of the TNSA method has been elucidated, we propose a simple method of achieving ultra-high brightness ion beams by creating a ion lens. Specifically, by curving the back of the surface of the target (concave), one can imagine focusing the ions to a point in space. We present 2-D simulations showing that this is possible, reaching particle intensities of $\sim 10^{18} \mathrm{~W} / \mathrm{cm}^{2}$ in a spot $1 / 3$ of a micron with $30 \mathrm{MeV}$ protons. Should this ion lens be realized in the laboratory, a large number of interesting and useful applications, including ion implantation of wafers, intense injectors to accelerators ${ }^{5}$, medical beams, and fast ignition ${ }^{6}$ would be possible.

\section{BACKGROUND}

The physical picture we propose is quite simple. It is based on a picture that arose 20 years ago during the Los Alamos Helios laser program, modified somewhat because of differences in target geometry and laser pulse length. Details can be found in Gitomer, et al. ${ }^{7}$ The first point to discuss is that they quickly realized that the majority of the ions detected with their spectrometer were protons, independent of target material. The cause for this was traced to a thin ( 50 Angstroms) layer of contaminant, which is always present in the experiments, a large fraction of which are hydrogen atoms. This was attributed to either ever-present water vapor in the target chamber, or a hydro carbon layer due to the vacuum pump oil. By heating the target to near-melting, they were able to eliminate the proton signal on aluminum and gold targets. 
Given the presence of hydrogen on the surface, they then explained the acceleration of the resultant ions as follows. The laser created very hot electrons, scaling roughly as predicted from resonance absorption, whose temperature they diagnosed by measuring the X-rays. To summarize, they found that the bulk ion energy scaled as. ${ }^{7}$ :

$$
E_{\text {ion }}=45 T_{\text {hot }}
$$

where $\mathrm{T}_{\text {hot }}$ is the hot electron temperature inferred form the measured X-rays. At their highest intensities, they measured ions up to $5.5 \mathrm{MeV}$. This corresponds to $100 \mathrm{keV}$ electron temperatures, which were also measured at these intensities. Note that they also observed a sharp ion cut-off in the ion energy spectrum. (In fact, this is also an obvious feature in the Rutherford ion spectrum, as reported by Fews, et. al. . ${ }^{8}$ (PRL). ) Gitomer et. al. were able to explain their data with 5 separate (although related) models. The most likely model to have relevance to our work was a simple analytic model of an isothermal rarefaction, followed by a free expansion. This essentially assumes that the ions were accelerated in the presence of a population of hot electrons, while the laser pulse was present (about a nanosecond). Then, the ions just free-streamed the majority of the rest of the distance to the detector. The scaling is quite simple: The ions gain energy proportional to the electron temperature,

$$
E_{\text {ion }}=\alpha T_{\text {hot }}
$$

where $\alpha$ is somewhere between 2 and 12, depending on the model. Note that they used spherical targets, which were about $100 \mu \mathrm{m}$ radius. Before we get too far into the calculations for our case, consider that if we have about a $\mathrm{T}_{\text {hot }} \sim 5 \mathrm{MeV}$, we would get a mean ion energy of about $22.5 \mathrm{MeV}$, in reasonable agreement with what was measured from the ion spectrometer. The cut-off energy is usually about 10 times the $\mathrm{T}_{\text {hot }}$, which for us would be about $50 \mathrm{MeV}$, a bit higher than Snavely and Singh's data for energetic ions. (This scaling also seems to hold for the Fews data.)

We will now briefly summarize the freely expanding plasma model, following Denavit ${ }^{9}$ as it can easily be extended to our case, as will be seen below. Although we begin with several assumptions, the zeroth order effects is seen by using this common model for the electron-ion interaction. We start with the expansion of a plasma into vacuum. We assume the expansion is 1-D in nature, corresponding to planar expansion. To obtain the density and velocity profiles of the expanding ions, we start with the continuity equation and the ion equation of motion. The electrons are assumed to be in isothermal equilibrium, and the electron density $n_{e}$ is given by the Boltzmann relation. If quasineutrality is assumed $\left(n_{e}=Z n_{i}\right)$, and electron inertia is neglected, one obtains:

$$
\frac{f n_{i}}{f t}+\frac{f\left(n_{i} v_{i}\right)}{f x}=0
$$




$$
\frac{f v_{i}}{f t}+v_{i} \frac{f v_{i}}{f x}=-C_{s} \frac{d n_{i}}{d x}
$$

where $v_{i}$ is the ion velocity, $C_{s}=\sqrt{Z T_{e} / M_{i}}$ is the ion sound speed, $T_{e}$ is the electron temperature, and $M_{i}$ is the ion mass. One obtains the self-similar solution by assuming that the solutions (i.e., $n_{i}$ and $v_{i}$ ) have a dependence on space and time of the form $\xi=x / t$. This gives the usual solutions:

$$
\begin{gathered}
n_{i}=n_{o} e^{\left[-\left(1+x / C_{s} t\right)\right]} \\
v_{i}=C_{s}+x / t .
\end{gathered}
$$

The electric field responsible for accelerating the ions is easily found from the electron equation of motion near the ion front:

$$
n_{e} e E=-\frac{f p_{e}}{f x}
$$

This accelerating field is

$$
E=\frac{T_{e}}{e C_{s} t}
$$

or (noting that for the self-similar solution, $C_{s} t=L_{n}$, where $L_{n}$ is the local plasma scale length)

$$
E=\frac{T_{e}}{e L_{n}}
$$

Notice that there are several problems with these solutions, namely various quantities going to infinity at early times and late times. These problems are resolved, once more realistic physics is included. For now, note that the electric field is large for high electron temperatures, but decreases with time. Also, as the scalelength of the plasma increase, the accelerating field decreases. This occurs on a picosecond time scale for relativistic electron temperatures. Additionally, the ion velocity increases with time, but the number of ions at the front (ie, the most energetic ions) decreases with time. In reality, the ions really don't get accelerated to infinity, because of two effects. First, as Denavit points out the electrons have finite inertia, and the ions eventually catch up. In fact, they catch up even more quickly, since the plasma out near the front is actually cooling (ie, it's not really isothermal). Kishimoto, Mima, Watanabe, and Nishikawa pointed out another important effect in $1983^{10}$ is the truncation of the electron Maxwellian at a finite energy. They found that this was an effective way to limit the maximum ion energy attainable near the front (where the ions are most energetic.) In fact they showed that the terminal velocity of the ion front position could be predicted by setting the local electron Debye length equal to the local scale length. From this argument, they estimated, for their parameters, the maximum ion velocity to be ${ }^{10}$ :

$$
\frac{v_{i}}{C_{h}}=2 \ln \left(\omega_{p i 0} t\right)+1
$$


Where $C_{h}=\left(T_{h} / M_{i}\right)^{1 / 2}$, and $\omega_{p i 0}=\left(4 \pi n_{o} e^{2} / M\right)^{1 / 2}$ are the hot sound speed and local ion plasma frequency, respectively. After this time, the ions could no longer accelerate, and thus would reach their terminal velocity. They reasoned that the electrostatic sheath at the ion front became weaker with time, eventually disappearing once the ion front coincides with the electron front. There are other effects, such as the fact that there are basically two disparate electron temperatures present ${ }^{11}$ on the back surface of the target, that tend to limit the ion velocity as well. As shown below, for the case of ultra-intense lasers, the cooling of the hot electrons and increase in the scale length are the two main contributors to the limitations on the maximum ion energy.

\section{SIMPLE MODEL}

Our case modifies this simple picture in the following trivial way. The scaling for $\mathrm{T}_{\text {hot }}$ is now given more by the ponderomotive potential, instead of by resonance absorption ${ }^{12}$. This energy spectrum has been shown to resemble a relativistic Maxwellian distribution with an effective temperature,

$$
T_{h o t} \cong m c^{2}-\frac{2 U_{p}}{m c^{2}} \sqrt{ }{ }^{1 / 2}
$$

which scales with the ponderomotive potential of the laser, $\mathrm{U}_{\mathrm{p}}[\mathrm{eV}]=9.33 \times 10^{-14} \mathrm{I}\left[\mathrm{W} / \mathrm{cm}^{2}\right] \lambda^{2}\left[\mu \mathrm{m}^{2}\right] .{ }^{13}$ Note that the acceleration mechanism is not only due to $\mathrm{JxB}$. For our Petawatt laser, interactions in the underdense plasma can give energies that are something like a couple times the ponderomotive potential. Hoever, this scaling was experimentally observed on the previous one micron lasers operating at about $10^{19} \mathrm{~W} / \mathrm{cm}^{2} .{ }^{14} \mathrm{We}$ now have enough to form a simple picture of our interaction. As shown in Figure 1, the front of the target expands spherically, as a plasma due to the interaction with the prepulse. The main laser pulse comes in, hits this plasma and the critical surface, generating a large number of hot electrons (the electron cloud.) Since the target is transparent to these energetic electrons, the cloud extends past the back of the target, whereupon it ionizes, then accelerates the proton layer on the back of the target. Estimates for the numbers and energies of protons follow, as does a discussion of the ion acceleration mechanism. 


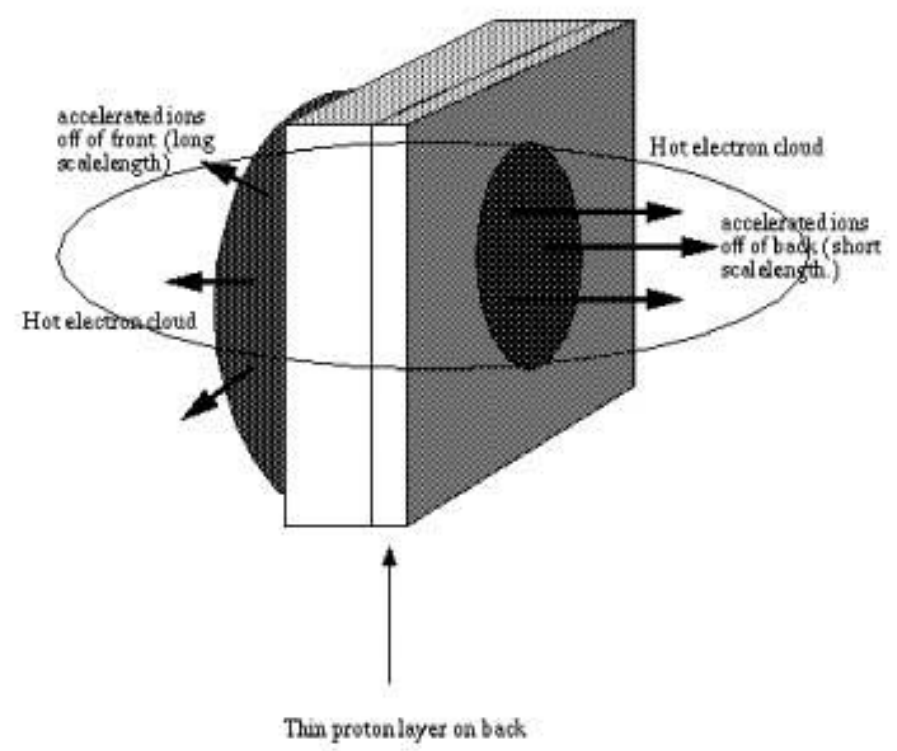

Fig, 1. Physical picture of the ion scceleration foe ultra-intense, ultra-shoet loser pulse, for thim torgets.

Fig. 1. Physical picture of the ion acceleration for an ultra-intense, ultra-short laser pulse interacting with a thin target.

In order to get an estimate for our experiment, we must use our best guess for the electron spectrum. This has been measured directly by T. Cowan, et al. ${ }^{4}$ However, note that the actual electron spectrum in the target may have be different than that actually measured. Although some of the first electrons escape the target before much potential is built up in the target, many of the lower energy electrons lose their energy on the way to the detector. This is because the electrons lose energy to the ions as they go from the target to the detector. We will use a $\mathrm{T}_{\text {hot }}$ of $7 \mathrm{MeV}$ as a first estimate. Note that if we use the Helios scaling, the maximum ion energy should be about $70 \mathrm{MeV}$, slightly higher than that measured by Snavely et. al. ${ }^{3}$. From the equation above, which gives the accelerating electric field, we see that it is both a function of electron temperature and density at the ion front. This makes it somewhat difficult to make an estimate for our experimental parameters, since we do not know either of these numbers. What we do know, though, is the maximum ion energy, and the total energy in the ions. By taking reasonable values for the density and temperature of the hot electrons, we can then estimate theses two unknown parameters. First, take the maximum ion energy to be $40 \mathrm{MeV}$, and the ion energy to be about 30 Joules. We now begin a string of assumptions. First, we assume that the absorption into the electrons was about 30 percent of the incident energy. This is 200 Joules. We assume that the direct absorption of laser light into the ions is of the order of a couple per cent. We know most of the energy in electrons ends up in the ions. If we assume the electron cloud envelopes the target (similar to the Los Alamos experiment), then if the target is a cube, 
each side of the cube will end up with about 1/6 the energy of the hot electrons. This is about 30 Joules. This implies that the amount of electron energy that goes into ions heading out the back of the target is about 30 Joules, as measured experimentally. Now assume that the average energy of the ions is $20 \mathrm{MeV}$. Equate the total energy with $\mathrm{N}_{\text {ion }} * \mathrm{E}_{\text {ave }}$ to find that we should get about $10^{13}$ protons. Assume the thickness of the proton layer is 100 Angstroms, and that the back of the target is about _ mm. Then we find that there are about $10^{18}$ protons available: more than enough. This model would say that roughly the same amount of ions would come off all the other sides just like they come off the back, except the front, which is a special case, due to the large prepulse of the petawatt.

Now, what kind of accelerating gradient can get ions up to these kinds of energies? We find that it, as distinct from previous studies, we get markedly different accelerating E-fields, depending strongly on the ion density scale length. This point is important in understanding the difference in ion energies between the front and back of the target. In fact, it is also important in understanding the directionality of the ions. To summarize, we find that for short density scale lengths, like those found on the back of out targets, the accelerating electric field can reach extremely high values (tens of $\mathrm{MeVs}$ per micron). It is important to note that this state can only be achieved with an ultra-short pulse creating a large number of electrons in a time short compared to a sound speed that can get to an unperturbed, sharp density surface (like the back of a thin target.) On the front of the target, where the prepulse has created a scale length of the order of 100 's of microns, the accelerating gradient is much less, since the accelerating force on the outermost ions is

$$
m_{i} \frac{d v_{i}}{d t}=\frac{T_{e}}{n_{i}} \frac{f n_{e}}{f x} \cup \frac{T_{e}}{L_{n}},
$$

where quasineutrality is assumed, and the scale length is defined as usual. Briefly, this equation says that for a quickly-created hot electron cloud that lasts a finite amount of time (same for front and back) we can expect that the ions on the front of the target (where the preformed plasma is large) to be less energetic than the ions on the back of the target. The ions off the back can be very energetic. In fact, they can attain energies in a way that was not possible before the advent of ultra-intense, ultra-short pulse lasers.

The second important point is that of directionality. Numerous hydro calculations of the prepulse on the petawatt have shown that the blow-off is quite three dimensional. That is, it is 3-D the sense that a sphere of blow-off plasma, with a radius of 50-100's of microns, is present before the main pulse (which creates the hot electron cloud) hits the target. Since our proposed model predicts quite fast ion acceleration in the first 1/10-10 picoseconds after the main pulse hits, the curvature of the accelerating surface, at the time the fast electrons are created, is clearly crucial to determining the directionality, as discussed below. 


\section{SIMULATION RESULTS}

We now present simulation results of a two component plasma: one hot electron component, and a cold ion component. We have also completed numerous three component simulations, with a fraction of hot electrons, a fraction of cold electrons, and a fraction of ions. In addition, we have also done four component cases, where we have hot electrons, cold electrons, and protons on the surface of a heavier ion underneath the protons. Although the latter two cases are more representative of our experimental conditions, the physics of the all three cases are qualitatively the same. The two component case is less complicated, since issues of fractions of hot versus cold electrons is not present, and we can isolate the ion acceleration dependence on electron temperature and scale length. The 3 component results will be reported elsewhere, although suffice it to say that, as expected, the acceleration gradient decreases, with decreasing fraction of hot electrons.

The basic set-up for the simulations is this: Start with a 1-D slab of plasma of width $\mathrm{L}_{\mathrm{p}}(\sim 2-20 \mu \mathrm{m})$, at some density $n_{p e}=n_{p i}(=10$ for most of the cases presented here). Further, the ions are taken to be cold protons $\left(\mathrm{m}_{\mathrm{i}} / \mathrm{m}_{\mathrm{e}}=1836\right)$, and the electrons are taken to have a quasi-Maxwellian temperature of between $100-500 \mathrm{keV}$. The slab of plasma is in contact with the right side of the simulation box. Particles leaving out this boundary are reflected back into the box. On the right side of the plasma slab is vacuum, extending well beyond the (initial) slab boundary, usually tens of microns. Thus, the ions expand out into vacuum on the right side of the slab. The density at the right side of the slab is always linearly ramped from $10 \mathrm{n}_{\mathrm{cr}}$ to zero. We will present results from two cases. First, where the density has been ramped from $10 \mathrm{n}_{\mathrm{cr}}$ to 0 over 0.05 microns, and secondly, where the density has been ramped from $10 \mathrm{n}_{\mathrm{cr}}$ to 0 over 10 microns. 


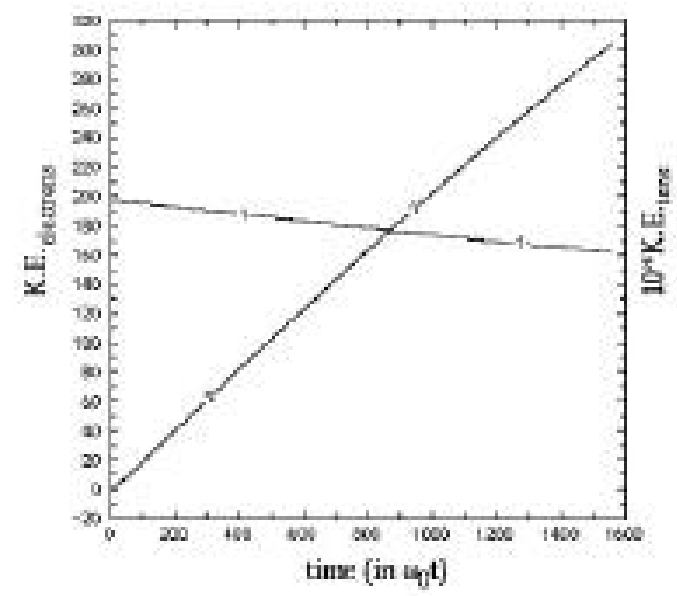

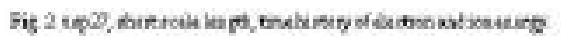

The first example will be the simple case of a slab of plasma with initial conditions: $\mathrm{T}_{\mathrm{e}}=500 \mathrm{keV}$, $\mathrm{n}_{\mathrm{e}}=\mathrm{n}_{\mathrm{i}}=10 \mathrm{n}_{\mathrm{cr}}, \mathrm{L}_{\mathrm{p}}=9$ microns, and the plasma has a sharp ramp in density over only 0.05 microns. This is representative of the backside of either the thin $\mathrm{CH}$ or thin $\mathrm{Au}$ (since the proton layer is present on surface) experiments. The initial electron kinetic energy, in code normalized units, is 198, and the ion energy is essentially 0 . After a time $\omega_{0} t=1600$, we find that the electrons have lost $20 \%$ of their energy to ions that now possess about 30 energy units, with the remainder residing in the electrostatic field (Fig. 2: note the ion energy scale has been multiplied by 10). The most energetic ion has an energy of $6.5 \mathrm{MeV}$, at a time of $\omega_{0} t=1600$. A history of the ions being accelerated, for times up to about $\omega_{0} t=1000$ is shown in Fig. 3(a). Figure 3(b) shows the actual ion spectrum at the same time, showing a relatively flat highenergy component, and a cut-off at $5 \mathrm{MeV}$. It is clear that most of the acceleration of the protons occurred in a very short distance ( $<3$ microns) as expected. This fact can be explained by looking at a snapshot of the accelerating electric field at an early time $\left(\omega_{0} \mathrm{t}=130\right)$ in Figure 4.
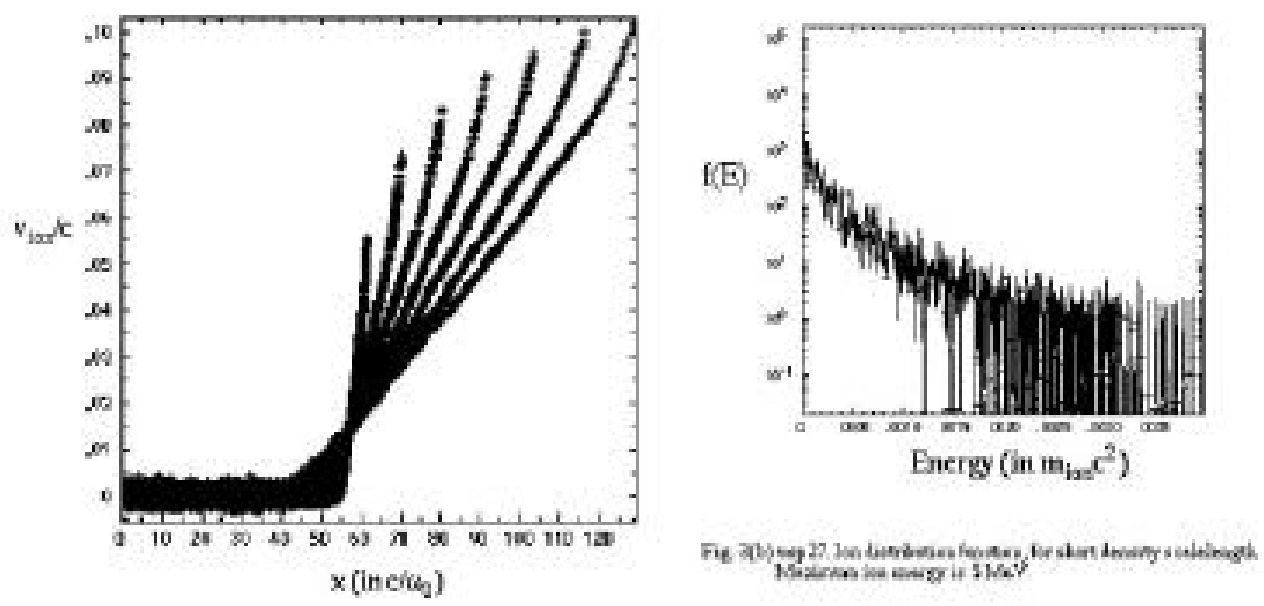


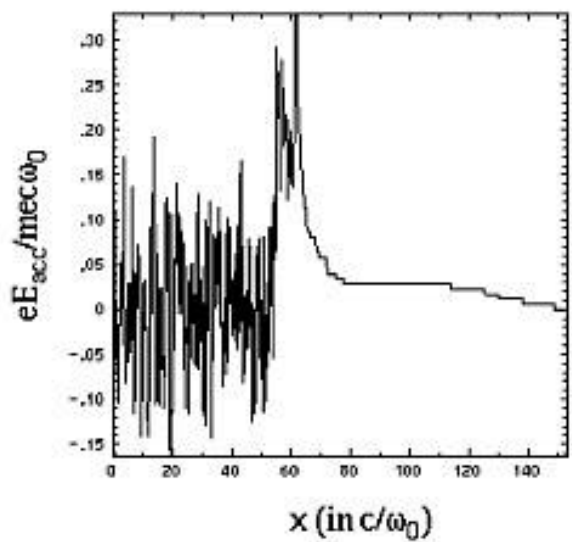

Fig. 4. Accelerating eleccric field, ar early ưme $\left(w_{-} \mathrm{Ot}=130\right)$

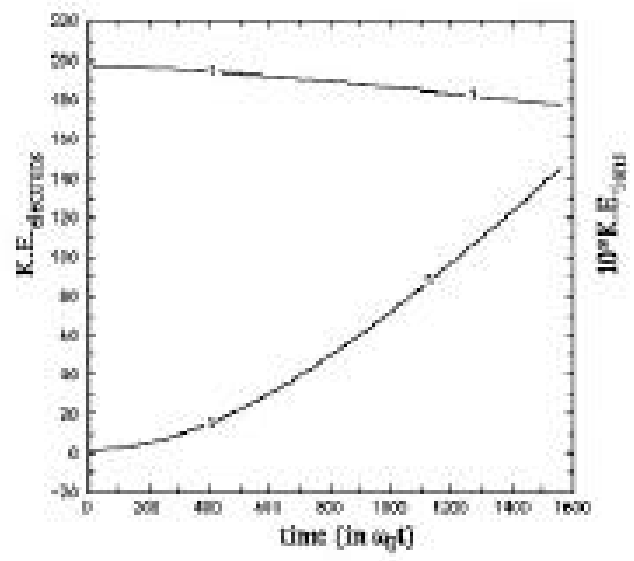

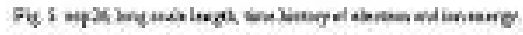

We now look at a nearly identical case, except now, the plasma slab is only 2 microns wide, but goes from $10 \mathrm{n}_{\text {cr }}$ to 0 over 14 microns. This is somewhat representative, at least qualitatively, to the case on the front of the target, where there exists a finite scale length. The number and temperature of the electrons is the same as the case above. Fig. 5 shows the electron and ion energy histories. Here, we see that the ions gain much less energy (about_) than they did in the sharp density gradient case, over an identical time period. More dramatically, we see the difference when one compares the maximum ion energies achieved in the two cases. We find that the maximum ion velocity in the run is $1.1 \mathrm{MeV}$ (compared to 6.5 in the sharp density case). A history of the early time ion velocities is shown in Fig. 6(a), with a representative spectrum show in Fig. 6(b). One point of interest is that the accelerating electric field in both cases, for late times, approaches the same value $\left(e E_{a c c} / c \omega_{0} m_{e} \cup 005\right)$. However, for early times in the long density scale length case, we see that the accelerating field is about _ of what it was for the sharp density case. Other simulations with higher temperatures give qualitatively same results. For comparison with Fig. 4 , a snapshot of the accelerating electric field at an early time $\left(\omega_{0} t=130\right)$ is shown in Figure 7 . As expected, it is considerably lower than the corresponding field in Fig. 4. 


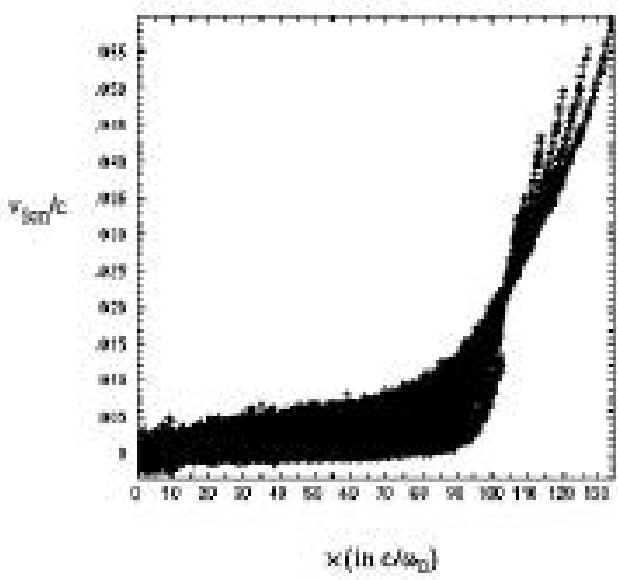

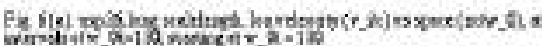

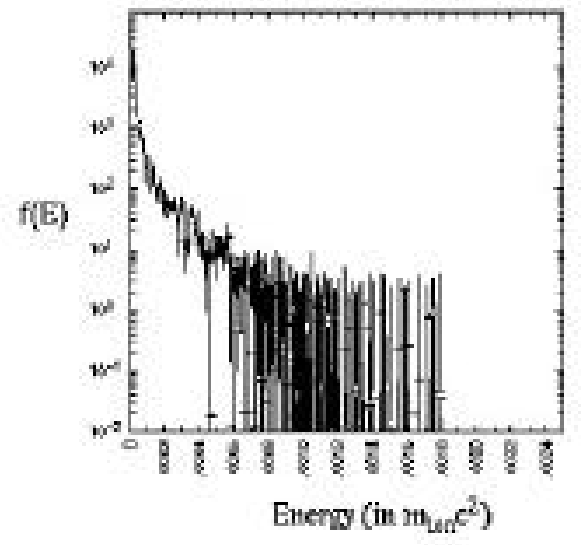

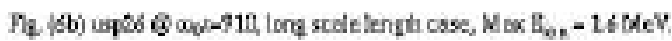

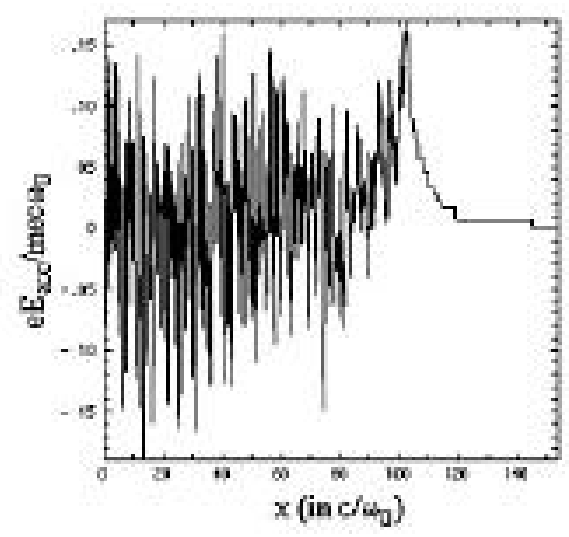

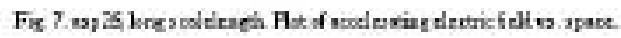

\section{2-D SIMULATION}

From the analysis above, we can assume that the accelerating E-field is strongly dependent on the spatial distribution of the electrons that reach the rear of the target. We now present a series of 2-D simulations that were done in order to try and understand the angular dependence of the ions ejected from the back surface. From nuclear activation experiments, it is found that the electron "jet" through the target is consistently found in a half angle of approximately 50 degrees ${ }^{15}$. Although we cannot simulate the exact experimental parameters for lack of computer memory, we can attempt to understand the effects associated with spatially varying electron energy in the transverse dimension. To this end, in a simulation box of width 25 microns, and length 10 microns, we strike a facsimile of a solid target (a $25 \mathrm{n}_{\text {cr }}$ plasma) with a 3 micron FWHM, $40 \mathrm{fSec}, \mathrm{I} \lambda^{2}=4 \times 10^{20} \mathrm{~W} / \mathrm{cm}^{2}, 1$ micron laser (Fig. 8). There is a slight ramp on 
the front of the target ( 0 to $25 \mathrm{n}_{\mathrm{cr}}$ over 1 micron). The scale length on the back of the target is extremely small: $25 \mathrm{n}_{\mathrm{cr}}$ to 0 in a distance of 0.01 microns. This configuration achieves our goal of creating an electron distribution that is spatially varying in the transverse (y) dimension, as shown in Fig. 9. In order to simulate this effect in our limited simulation box (Fig. 8), although not exactly what is believed to be present in the petawatt experiments.

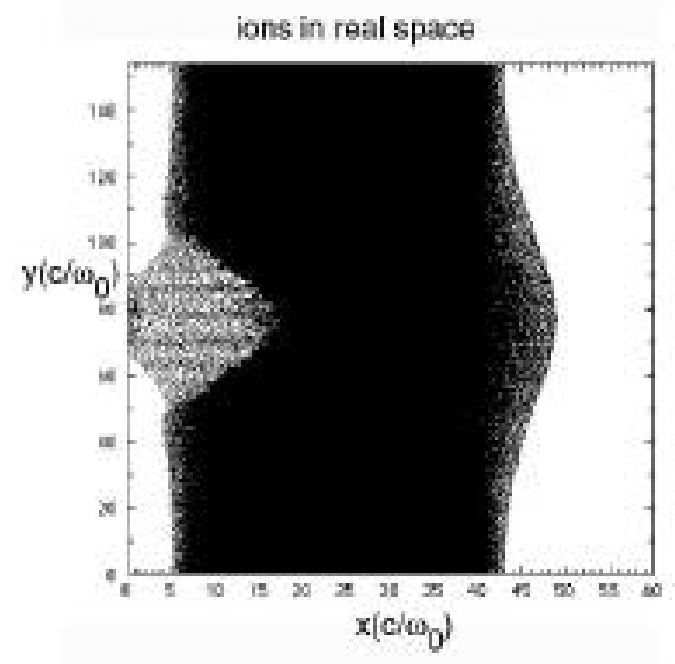

Fig. 8 Real space for the 2-D simulation.

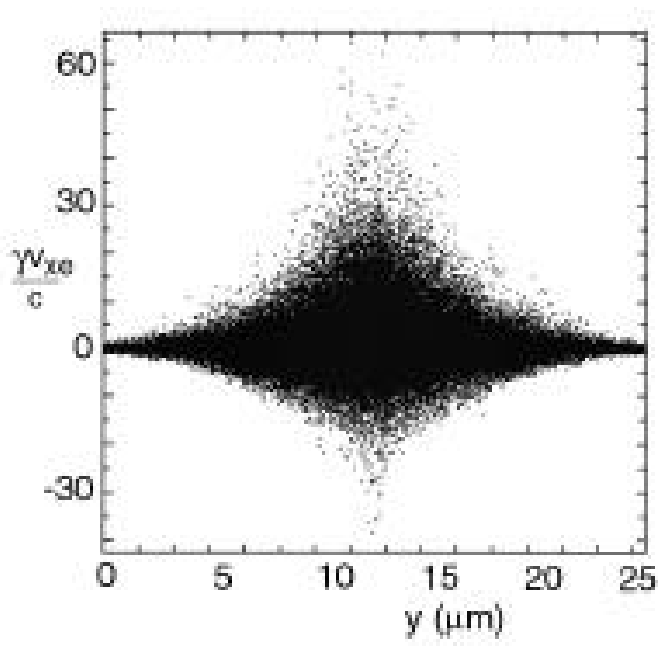

Fig. 9 Electron momenta as a function of radial position.

The resulting ion distribution in phase space, after a time of $1 \mathrm{ps}$, is shown in Fig. 10. There are essentially 2 components here. The ions going off at angles (roughly 30 degrees) are entirely from the front of the target, while the large spike from $\mathrm{v}_{\mathrm{x}} / \mathrm{c}=0$ to 0.2 is due to ions off the back. It is clear that there is a variation induced in the ions off the back. In particular, the higher energy ions have a noticably larger transverse velocity than lower energy ions. This can be attributed to the fact that, during the initial, large acceleration phase of the ions, the back surface is becoming curved outward, such that the ions on axis are moving fast (since the number of hot electrons is highest on axis, as seen in Fig. 9) whereas those off axis are slower. As this continues, the accelerating field is no longer planar, but becomes 3 dimensional. Thus, the elctrostatic field that is initally accelerating the ions ends up with a substantial radial component, which acts to increase the radial size of the beam, over and above what one might expect from a purely planar, laminar flow of protons from the back. In fact, at distances far from the back of the target, if one were to follow the trajectories back to get an estimate of the spot size, it is quite possible that one would underestimate the true spot size of the proton beam on the target surface by a substantial amount. 


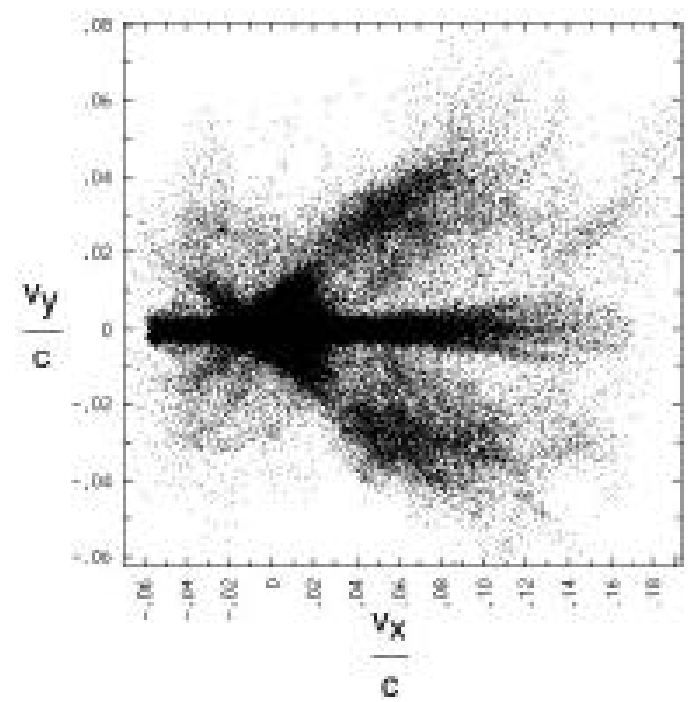

Fig. 10. Ion velocity phase space, showing ions from front of target (the "lobes" at an angle) and the ions off the back (the fast ions with larger positive $\mathrm{v}_{\mathrm{x}}$, and small transverse velocities.)

\section{ION LENS SIMULATION}

As seen above, the ions are accelerated in a very short time $(\sim$ psec $)$ over a very short distance $(\sim 10$ microns) to high energies $(\sim 10-50 \mathrm{MeV})$. We have also seen that the acceleration is parallel to the normal of the back of the target, for those ions that start at the back of the target. We now extend this, by considering a curved surface on the back of the target. For example, consider a concave surface, as shown in Fig. 11. We now irradiate the left side of the slab with a $50 \mathrm{fSec}, 4 \times 10^{20} \mathrm{~W} / \mathrm{cm}^{2}, 40$ micron wide laser pulse. As time continues, we find that the ions on the inside of the half circle are accelerated toward the focus of the circle, chosen, in this case, to be at $\mathrm{x}=70 \mathrm{c} / \mathrm{w}_{0}$. The last frame of Fig. 11 shows the ion real space at a time when the ions from the surface are converged on the focal spot for this "lens". We find that the FWHM of the ion "spot" is 1/3 micron, as shown in Fig. 12. The average energy of the ions at this time is about $20 \mathrm{MeV}$. For the density achieved, we find that the beam intensity reaches about $3 \times 10^{18}$ $\mathrm{W} / \mathrm{cm}^{2}$.

An important aspect of generating proton beams with this method is the ability to both propagate this large current over relatively long distances, and focus to tiny spots. An important reason that this can occur is the fact that because the electrons, which caused the acceleration of the ions in the first place, are already present (although they have lost most of their energy early on, as shown above) the ion "beam" is space-charge neutralized. Therefore, the beam can propagate due to the presence of electrons. 


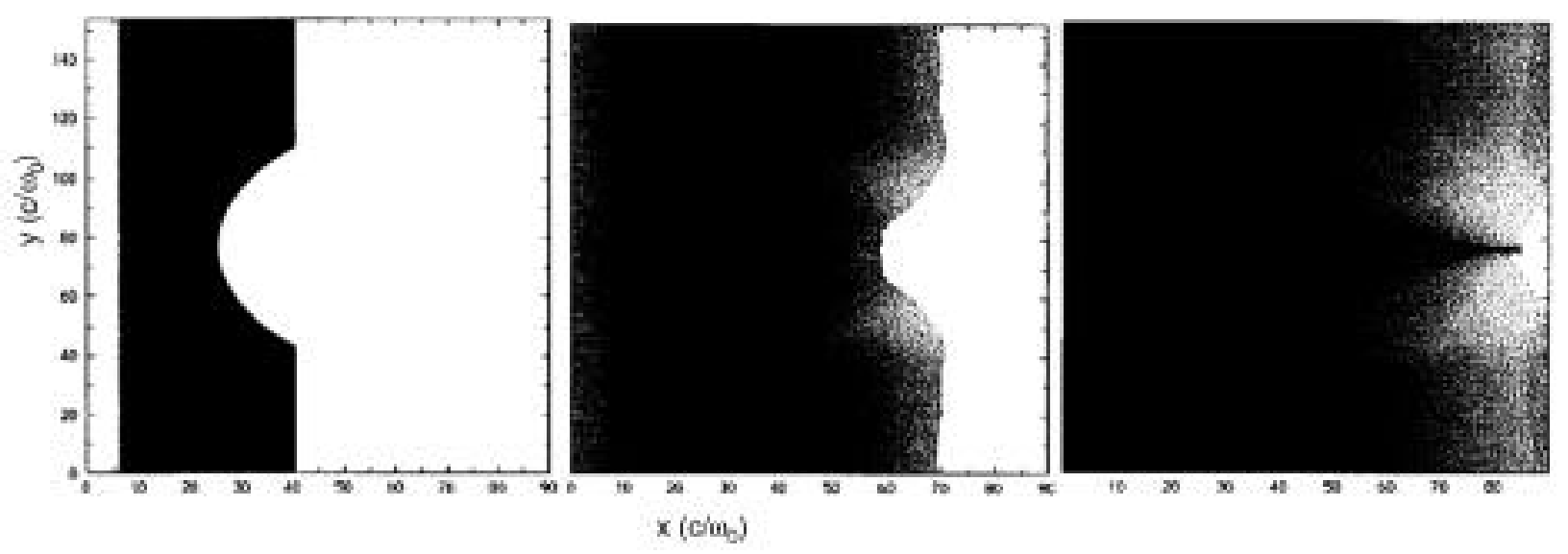

Fig. 11. Ion lens formed by shaping the back of a solid target. Time evolution of the ions focusing to a point behind the target.

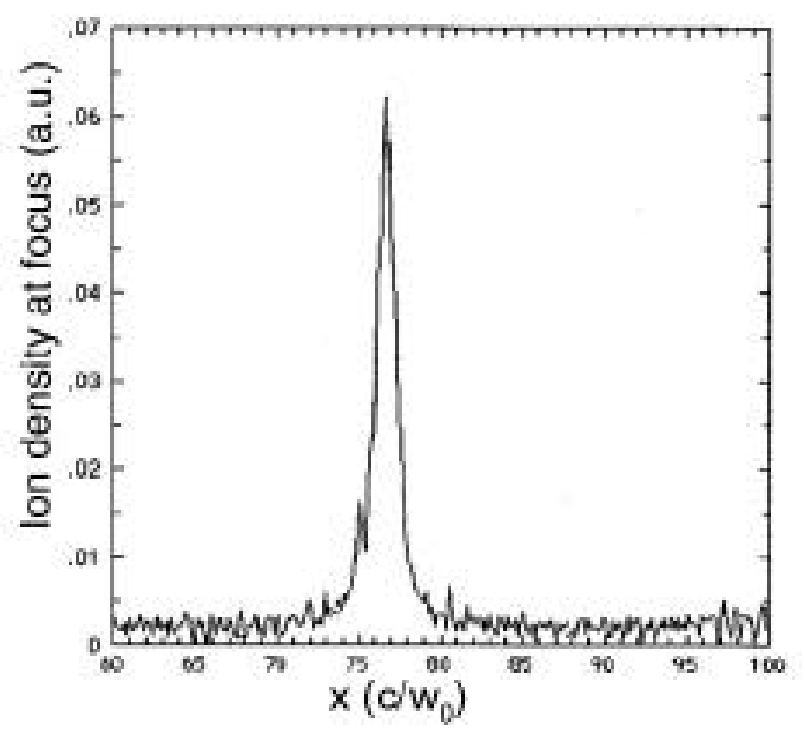

Fig. 12. A slice through the ion density near the focus.

\section{CONCLUSION}

We have presented a model of ion acceleration (i.e. proton acceleration) observed in the petawatt experiments. Briefly, as shown in Figure 1, the prepulse creates large plasma in front of a solid target. Once the main pulse hits the target, a cloud of energetic electrons (1-10 MeV in effective temperature) is generated, which extends past the ions on both the front and back of the target. Since the protons on the back are in a sharp, flat density gradient, they are accelerated quickly (in the first few microns off the target) to high energies in the forward direction (since the surface, locally, everywhere) is flat. On the front, the outermost ions are in a sphere, in a long scale length plasma (due to prepulse) and therefore are accelerated to lower energies, and are spread out into $2 \pi$ steradians. 
As a final point, we consider the ramifications of this model. To choose the energy of the ions, once chooses the intensity of the laser, and hence the energy of the electrons. The target must be thick enough such that the back surface remains flat and unperturbed by the prepulse. The hot electrons must be created in less than a picosecond to prevent "preheat, and thus a lengthening of the scale length at the back of the target, before the bulk of the hot electrons are produced. Since the majority of the acceleration of the ions off the back of the target happens in a short time ( $<10$ picoseconds) and distance $(<10$ microns) from the target surface, local curvature of the back of the target could play a role in the directionality and focusability of the resulting proton beam. Thus, if the ion acceleration truly is normal to the target surface, it may be possible to create a sort of solid density "ion lens", by curving the target back appropriately, as shown in Figure 11.

To conclude with the comparision to the petawatt experiments reported by Snavely, in this manuscript, we have only presented a physical picture as to what is happening in the actual experiments which is consistent with trends in the experimentally measured quantities. Computer simulation today is limited in the size of system, density of plasma, collisional effects, and length of time that one can simulate. Thus, we cannot perform the exact petawatt experiment, with its nanosecond long pedestal, and it's $100 \mu \mathrm{m}$ scale length and effective spot size in even two dimensions. What we have done is study the most dominant physical regime (collisionless plasma, for the hot electrons, since the energy loss due to $\mathrm{dE} / \mathrm{dx}$ and radiation are negligible for multi-MeV electrons), note the trends, and scale to the experiment appropriately.

As an example experiment to test this hypothesis that is both realistic and readily comparible to the parameter space investigated in this paper, assume that one has a laser that can provide 15 Joules in 100 fsec. Maximize the prepulse, so that a large, preformed plasma can be created. Come in at an angle of incidence so as to maximize coupling to the hot electrons via resonance absorption and Brunel absorption. Use a radius of 22 microns, so that an intensity of $10^{19} \mathrm{~W} / \mathrm{cm}^{2}$ is attained. This will produce electrons around 1-3 MeV. Assume that 1/3 the energy goes into the electrons, and that $1 / 3$ of this energy goes into ions out the back, with an average energy of 5-10 MeV. This gives somewhere around $10^{12}$ protons, easily detectable by wither radiochromic film or the Cowan and Roth magnetic spectrometer.

To conclude, the ion acceleration mechanism is the result of a population of hot electrons (generated in the blow-off plasma created by the laser prepulse interacting with the front of the target) that go through the target and ionize the proton monolayer on the back of the target. Although the electron temperature, 
$\mathrm{T}_{\text {hot }}$, and the ion scale length, $\mathrm{L}_{\mathrm{n}}$, rapidly change with time, one can get an estimate for the accelerating fields generated by assuming they are constants during the time the laser pulse is on and is generating hot electrons. By applying Poisson's equation and assuming a Boltzmann distribution, one obtains the results of the standard self-similar plasma expansion model, which says that for an electron temperature of $\mathrm{T}_{\text {hot }}$, the electric field acting on the ions is given by $\mathrm{E}=\mathrm{T}_{\text {hot }} / \mathrm{eL}_{\mathrm{n}}{ }^{9}$. This shows there will be much stronger acceleration at the sharp density interface on the back of the target than on the front, where the scale length is hundreds of microns. This results in a rate of energy transfer to the ions which is initially much greater on the back of the target. If the hot electrons dissipate their energy rapidly, the result will be a large energy transfer to the ions at the rear surface. Previous experimental measurements of hot electron temperatures using ultra-intense lasers has shown that for our parameters the hot electron energies are of order $\mathrm{MeV}^{\prime} \mathrm{s}^{14}$. Since the initial scale length on the back of the target is roughly a micron, we see that initially electric fields of $\mathrm{MeV} /$ micron are possible. This model, modified by the fact that there are two electron temperatures present ${ }^{11}$, is consistent with the facts that (1) the ions are observed normal to the back of the target, and (2) protons with energies of tens of MeV's are observed. 2-D Particle-In-Cell simulations have confirmed these findings.

We would like to acknowledge useful discussions with the entire petawatt team. SCW would like to thank John Zonfrello for computer assistance.

This work was performed under the auspices of the U.S. Department of Energy by the Lawrence Livermore National Laboratory under Contract W-7405-Eng-48.

\section{REFERENCES}

1. M. Key et. al., to be published in Proc. $17^{\text {th }}$ IAEA Fusion Energy Conference (1999).

2. S. Hatchett, et. al., to be published, Physics of Plasmas, (2000).

3. R. Snavely, M. Key, S. Hatchett, T. E. Cowan, M. Roth, T. W. Phillips, M. A. Stoyer, E. A. Henry, T. C. Sangster, M. S. Singh, S. C. Wilks, A. MacKinnon, A. A. Offfenberger, D. M. Pennington, K. Yasuike, A. B. Langdon, B. F. Lasinski, M. D. Perry, E. M. Campbell, submitted to Phys. Rev. Lett. (1999).

4. M. Roth, et. al. to be submitted to Phys. Rev. Lett. (2000).

5. T. Cowan, et. al. to be submitted to Nature (2000); S. C. Wilks, T. Cowan, A. B. Langdon, M. Roth, and W. L. Kruer, to be submitted to PRL, (2000).

6. M. Roth, T. Cowan, M. Key, S. Hatchett, C. Brown, M. Christl, W. Fountain, J. Johnson, T. Parnell, D. Pennington, M. D. Perry, T. W. Phillips, R. Snavely, S. C. Wilks, K. Yasuike, to be submitted to Phys. Rev. Lett. (2000).

7. S. Gitomer, et. al., Phys. Fluids 29, 2679 (1986).

8. A. P. Fews, P. Norreys, F. N. Beg, A. R. Bell, A. E. Dangor, C. N. Danson, P. Lee, and S. J. Rose, Phys. Rev. Lett., 73, 1801 (1994). 
9. J. Denavit, Phys. Fluids 22, 1384 (1979).

10. Y. Kishimoto, K. Mima, T. Watanabe, and K. Nishikawa, Phys. Fluids 26, 2308 (1983).

11. L. M. Wickens, J. E Allen, and P. T. Rumsby, Phys. Rev. Lett. 41, 243 (1978);

12. S. C. Wilks, W. L. Kruer, M. Tabak, and A. B. Langdon, Phys. Rev. Lett. 69, 1383 (1992).

13. W.L. Kruer and K. Estabrook, Phys. Fluids, 28, 430 (1985).

14. K. Wharton, et. al., Phys. Rev. Lett. 81, 822, (1998).

15. M. Stoyer, et. al. , submitted to PRL (1999). 